\title{
TWO-RELATOR GROUPS WITH PRESCRIBED COHOMOLOGICAL DIMENSION
}

\author{
JAMES HOWIE \\ ABSTRACT. For each integer $n \geqslant 2$, an example is constructed of a 2-generator, \\ 2-relator presentation of a group $G(n)$ of cohomological dimension $n+1$.
}

This note is a response to the following question, which was put to me by J.-P. Serre.

Lyndon's Identity Theorem [3] implies that the cohomological dimension of a one-relator group is either $0,1,2$, or $\infty$. Is there a corresponding statement for two-relator groups?

It is very easy to construct two-relator groups of cohomological dimension $0,1,2$, 3 and $\infty$. Serre (unpublished) has constructed one of dimension 4, and Brown and Geoghegan [1] have shown that the two-relator group

$$
\left\langle x, y \mid\left[x, x^{y}\right],\left[x, x^{y^{2}}\right]\right\rangle
$$

is torsion-free, of type $F P_{\infty}$, and of cohomological dimension $\infty$. (Here $a^{b}$ denotes $b^{-1} a b$ and $[a, b]$ denotes $a^{-1} b^{-1} a b$.)

On the other hand, if there is a generator which does not appear in one relator, but essentially appears in the other, then the presentation is "reducible" in the sense of [2]. If in addition neither relator is a proper power modulo the other, then the group has cohomological dimension at most 2.

In fact, we construct examples of two-relator groups of any given finite cohomological dimension. Let $G(n)$ be the group

$$
\left\langle x, y \mid\left[x, x^{y}\right],\left[y^{n}, x\right] x^{y}\right\rangle .
$$

and $G(n)^{\prime}$ its commutator subgroup. Then $G(n) / G(n)^{\prime}$ is clearly infinite cyclic. We prove

Proposition. $G(n)^{\prime}$ is free abelian of rank $n$.

It follows that $G(n)$ is poly-(infinite cyclic) of Hirsch number (and hence cohomological dimension) $n+1$.

Received by the editors September 5, 1986.

1980 Mathematics Subject Classification (1985 Revision). Primary 20F05; Secondary 20F16.

Research supported by SERC Advanced Fellowship B/AF/630.

(C)1987 American Mathematical Society $0002-9939 / 87 \$ 1.00+\$ .25$ per page 
Proof of Proposition. Clearly $G(n)^{\prime}$ is the normal closure in $G(n)$ of $x$, so is generated by the elements $x_{i}=y^{-i} x y^{i}(i \in \mathbf{Z})$. Indeed the second defining relation can be rewritten in terms of these new generators as $x_{n}=x_{0} x_{1}$, so $G(n)^{\prime}$ is actually generated by $x_{0}, \ldots, x_{n-1}$, subject to the relations $\left[x_{i}, x_{i+1}\right](i \in \mathbf{Z})$.

Thus $G(n)^{\prime} / G(n)^{\prime \prime}$ is free abelian of rank $n$, and so it suffices to prove that $G(n)^{\prime \prime}=\{1\}$, in other words that $G(n)^{\prime}$ is abelian. To do this it suffices in turn (using the automorphisms $x_{i} \rightarrow x_{i+1}$ of $\left.G(n)^{\prime}\right)$ to prove that $\left[x_{0}, x_{i}\right]=1$ for all $i>0$, and we shall prove the latter statement by induction on $i$. Now $\left[x_{0}, x_{1}\right]=1$ by the defining relations, so suppose inductively that $\left[x_{0}, x_{i}\right]=1$ for all $i$ with $0 \leqslant i<k$. Then

$$
\left[x_{0}, x_{k-1}\right]=\left[x_{1}, x_{k-1}\right]=\left[x_{1}, x_{k}\right]=1 \text {, }
$$

so

$$
\left[x_{0}, x_{k}\right]=\left[x_{0} x_{1}, x_{k-1} x_{k}\right]=\left[x_{n}, x_{n+k-1}\right]=y^{-n}\left[x_{0}, x_{k-1}\right] y^{n}=1
$$

as claimed.

\section{REFERENCES}

1. K. S. Brown and R. Geoghegan, $F P_{\infty}$ groups and $H N N$ extensions, Bull. Amer. Math. Soc. (N.S.) 9 (1983), 227-229.

2. J. Howie, On locally indicable groups, Math. Z. 180 (1982), 445-461.

3. R. C. Lyndon, Cohomology theory of groups with a single defining relation, Ann. of Math. (2) 52 (1950), 650-665.

Department of Mathematics, University of Glasgow, Glasgow G12 8QW, Scotland

Current address: Department of Mathematics, Heriot-Watt University, Riccarton, Edinburgh EH14 4NS, Scotland 